\title{
Violation of contextual generalization of the Leggett-Garg inequality for recognition of ambiguous figures
}

\author{
Masanari Asanoํㅜ Takahisa Hashimoto ${ }^{2}$, Andrei Khrennikov ${ }^{3}$, \\ Masanori Ohya ${ }^{2}$, and Yoshiharu Tanaka ${ }^{2}$ \\ ${ }^{1}$ Liberal Arts Division, Tokuyama College of Technology,, \\ Gakuendai, Shunan, Yamaguchi 745-8585 Japan \\ ${ }^{2}$ Department of Information Sciences, Tokyo University of \\ Science, , Yamasaki 2641, Noda-shi, Chiba, 278-8510 Japan \\ ${ }^{3}$ International Center for Mathematical Modeling, in Physics, \\ Engineering, Economics, and Cognitive Science, Linnaeus \\ University, Växjö-Kalmar, Sweden
}

\begin{abstract}
We interpret the Leggett-Garg (LG) inequality as a kind of contextual probabilistic inequality in which one combines data collected in experiments performed for three different contexts. In the original version of the inequality these contexts have the temporal nature and they are given by three pairs of instances of time, $\left(t_{1}, t_{2}\right),\left(t_{2}, t_{3}\right),\left(t_{3}, t_{4}\right)$, where $t_{1}<t_{2}<t_{3}$. We generalize LG conditions of macroscopic realism and noninvasive measurability in the general contextual framework. Our formulation is done in the purely probabilistic terms: existence of the context independent joint probability distribution $P$ and the possibility to reconstruct the experimentally found marginal (two dimensional) probability distributions from the $P$. We derive an analog of the LG inequality, "contextual LG inequality", and use it as a test of "quantum-likeness" of statistical data collected in a series of experiments on recognition of ambiguous figures. In our experimental study the figure under recognition is the Schröder stair which is shown with rotations for different angles. Contexts are encoded by dynamics of rotations: clockwise, anticlockwise, and random. Our data demonstrated violation of the contextual LG inequality for some combinations of aforementioned contexts. Since in quantum theory and experiments with quantum physical systems this inequality is violated, e.g., in the form of the original LG-inequality, our result can be interpreted as a sign that the quantum(-like) models can provide a more adequate description of the data generated in the process of recognition of ambiguous figures.
\end{abstract}




\section{Introduction}

Mathematical modeling of the process of recognition of ambiguous figures is an intriguing problem which still has no completely satisfactory solution. Recently quantum(-like) models based on the mathematical formalism of quantum mechanics and its generalizations were applied to this problem [1]-12. As in any mathematical modeling project, the output of models has to be compared with results of experiments.

One of the basic intrinsically quantum probabilistic effects is violation of the formula of total probability which is experimentally exhibited in the interference effect [6]-8. In a series of papers [1]-3] such an effect was found in experimental data collected in sequential recognition of a pair of ambiguous figures. Then it was also found 4 that these data violate Bell's inequality [5].

Another quantum-like study on recognition of ambiguous figures was done by Atmanspacher et al. [10, 11] a quantum-like model of bistable perception. (A generalized quantum formalism was in use 12.) One of the important novelties in [10], 11] was application of temporal Bell inequalities, concretely the Leggett-Garg (LG) inequality 13.

Recently a quantum-like model of recognition of ambiguous figures was presented in the paper of Asano et al. 14]. The model matches very well with experimental data on determination of the structure of the Schröder stair which was shown with rotations for different angles.

In this paper we consider the possibility to use the inequalities of the LG type to check "quantum-likeness" of statistical data collected 14 in experimental studies on bistable perception. We interpret the LG inequality as a kind of contextual probabilistic inequality in which one combines data collected in experiments performed for three different contexts, cf. [9], 6], 10, [15], 8]. In the original version of the inequality these contexts have the temporal nature and they are given by three pairs of instances of time, $\left(t_{1}, t_{2}\right),\left(t_{2}, t_{3}\right),\left(t_{3}, t_{4}\right)$, where $t_{1}<t_{2}<t_{3}$. We generalize LG conditions of macroscopic realism and noninvasive measurability in the general contextual framework. Our formulation is done in purely probabilistic terms: existence of the context independent joint probability distribution $P$ and the possibility to reconstruct the experimentally found marginal (two dimensional) probability distributions from the $P$. We derive an analog of the LG inequality, "contextual LG inequality", and use it as a test of "quantum-likeness" of statistical data collected in a series of experiments on recognition of ambiguous figures. In our experimental study the figure under recognition is the Schröder stair 16] which is shown with rotations for different angles. Contexts are encoded by dynamics of rotations: clockwise, anticlockwise, and random. Our data demonstrated violation of the contextual LG inequality for some combinations of aforementioned contexts. Since in quantum theory and experiments with quantum physical systems this inequality is violated, e.g., in the form of the original LG-inequality (see, e.g., [17] and references hereby), our result can be interpreted as a sign that the quantum(-like) models can provide a more adequate description of the data generated in the process of recognition of ambiguous figures.

In probabilistic terms context dependence of (probabilistically deter- 
mined) mental states imply that the conventional model of probability theory, the Kolmogorov measure-theoretic model [18, cannot be applied to describe statistics of recognition of ambiguous figures. Thus our result on violation of the contextual LG inequality restricts the domain of applications of the Kolmogorov mode 1 . More general probabilistic models have to be tested, e.g., quantum probability and its generalizations [12], 6], 19]. Since neither physicists nor psychologists have a proper education in probability theory, at least in the axiomatic approach, we complete this paper with an extended appendix presenting both a brief introduction to the classical Kolmogorov model and a discussion on possible non-Kolmogorovian generalizations as well as relation to Bell type inequalities.

Contextuality of mental representations is one of the fundamental features of cognition. In particular, mental contextuality is one of the main motivations for applications of the quantum formalism to modeling of cognition 6, 19-24, 14 and more generally biological information processind 25], 26, since quantum mechanics is also fundamentally contextual. Typically quantum contextuality is expressed in the form of the KochenSpecker theorem. However, recently contextuality was also represented with the aid of Bell type inequalities, e.g., 27]. These recent theoretical and experimental results on the Bell type expression of contextuality match well with the contextual approach to the problem of violation of Bell's inequalities developed by one of the authors of this paper [9], 28][30], see also [31]-39]. Originally Bell mixed in one cocktail nonlocality and realism. The standard conclusion from violation of the inequalities of the CHSH-type is that "local realism" is incompatible with quantum behavior is not easy to interpret. What is the problem? Nonlocality? Realism? Both? The contextual viewpoint to violation of Bell type (so to say, spatial) inequalities helps a lot to clarify this problem. The contextual (spatial) Bell inequality is violated for a single particle, e.g., a neutron [27, therefore the problem of (non)locality can be automatically excluded from consideration. The same happens in the case of temporal contextual Bell inequalities, including the LG inequality. We shall come back to this discussion and extend it to the problem of inter-relation of mental contextuality and mental realism in sections 2.1 7. (We also remark that T. Nieuwenhuizen invented the terminology Contextuality Loophole summarizing outputs of studies [31, [9], 28- 30], 32-36].)

We point out that contextual dependent systems (in physics, biology, economics, finances, and social science) and non-Kolmogorov probability theory can be described mathematically [19], [8] by the adaptive dynamics and the operation of lifting (the latter is widely used in quantum information theory [40]).

It is important to remark that we consider contextuality in its the most general form, as N. Bohr [4] did: the whole experimental arrangement has to be taken into account. J. Bell [5] considered only a very special

\footnotetext{
${ }^{1}$ In fact, the real situation is more complicated, see section 8.4

${ }^{2}$ The formal identity of quantum-like models of "decision making" by cells and cognitive systems can be considered as an argument for recognition of existing of a kind of cell's cognition.
} 
form of contextuality: dependence of the result of measurement of some observable $A$ on joint measurement of another observable $B$ compatible with $A$. In cognitive science the situation is even trickier. Measurements are typically self-observations which the brain performs on itself. Therefore "the whole experimental arrangement" includes not only the "external experimental arrangemenet", e.g., prepared by researchers in cognitive psychology, but also "internal arrangement" including the brain state.

We remark that this paper has nothing to do with study of quantum physical processes in the brain. We proceed with the operational approach to quantum theory as a formalism describing in probabilistic terms measurements of in general incompatible observables. Such observables can be of any nature, physical, mental, biological [6, [19.

\section{Leggett-Garg inequality}

\subsection{Conditions of derivation}

At the beginning of the discussion in the paper [13], Leggett and Garg $(\mathrm{LG})$ postulated the following two assumptions:

(A1) Macroscopic realism: A macroscopic system with two or more macroscopically distinct states available to it will at all times be in one or the other of these states.

(A2) Noninvasive measurability: It is possible, in principle, to determine the state of the system without arbitrarily small perturbation on its subsequent dynamics.

Under these assumptions, the correlation functions must satisfy the LG inequality which will be presented in the next section. However, quantum mechanics violates the LG inequality as well as the same analogue of Bell's inequality or CHSH inequality. Therefore this violation means that at least one of the two assumptions fails for quantum systems.

Although in the derivation of the LG inequality, section 3 both conditions play important roles, their foundational value is different. The main issue is realism, whether one can still proceed with (A1), macroscopic realism, in the quantum world. Therefore the main part of the LG paper 13 is devoted to discussion about possible physical experimental schemes which may lead to noninvasive measurements or at least measurements in which invasiveness is small comparing with the degree of violation of the LG inequality. There are claims, e.g., the experiment in [17, that such negligibly invasive measurements were performed experimentally and the LG inequality was violated. This is often seen as an important argument in favor of non-objectivity of quantum observables.

However, the LG approach plays an important foundational role even if the possibility that measurements are non-negligibly invasive cannot be excluded. We know that classical systems and measurements on such systems satisfy conditions (A1) and (A2); e.g., airplane's trajectory. Therefore by violating LG we at least know that a phenomenon under study cannot be described classically. 
In cognitive science it is not easy (if possible at all) to come with an experimental scheme which would lead to (at least approximately) noninvasive measurements. The brain is a kind of self-measurement device, by giving an answer to a question the brain definitely perturbs its mental state and non-negligibly. And the introspective measurements have definitely the lowest degree of non-invasiveness. Therefore it seems that violations of the LG type inequalities for data collected, e.g., in cognitive psychology, cannot lead to the conclusion that mental realism is questionable. Here realism is understood in the sense of objectivity of mental observables, that their values can be assigned, say to the brain, a priori, i.e., before measurements. Nevertheless, such violations show that the data under consideration is nonclassical, i.e., it is not similar to data collected, e.g., from an ensemble of moving airplanes.

However, our main point is that in relation to the problem of cognition the standard physical viewpoint on conditions for derivation of the LG inequality, namely, the mixture of macrorealism and non-invasiveness, does not match so well with the mental situation. As was emphasized, the very notion of (non)invasive measurement loses its clearness for self-measuring devices and the brain is one of such devices. The paper advertises the contextual viewpoint on the mental phenomena developed in the series of works [6], 19]-24, [14. It seems that Bell type inequalities, including the temporal ones, can be used to distinguish contextual and non-contextual realism and more generally (since mental processes are fundamentally random) contextual and non-contextual probabilistic representations. As will be seen from coming presentation, non-contextuality of representation of probabilistic data implies constraints on such data, in the form of various inequalities. By using contextual representation a system (including the brain) can violate such constraints. We shall come back to this discussion in section 7

\section{Contextual viewpoint on the proof of LG inequality}

To provide to a reader the possibility to compare the original LG inequality with our contextual generalization, see section 4, and at the same time to add the contextual flavor to the LG approach, we present the original LG derivation by considering time as a context parameter.

Let $Q$ be an observable quantity which takes either +1 or -1 . In the original discussion by LG, $Q$ is the observable of position of a particle in the two potential wells. However we can discuss another two-level system, e.g. spin- $\frac{1}{2}$ system.

The measurement of the two-level system is perfomed on a single system at different times $t_{1}<t_{2}<t_{3}$. We denote the observable at time $t_{k}$ by $Q_{k}(k=1,2,3)$. By repeating a series of three measurements, we can estimate the value of correlation functions by

$$
C_{i j}=\frac{1}{N} \sum_{n=1}^{N} q_{i}^{(n)} q_{j}^{(n)},
$$


where $q_{i}^{(n)}$ (or $q_{j}^{(n)}$ ) is a result of the $n$-th measurement of $Q_{i}$ (or $Q_{j}$ ). Note that the correlation between $Q_{i}$ and $Q_{j}$ takes the maximum value $C_{i j}=1$ when $q_{i}^{(n)} q_{j}^{(n)}$ equals to 1 for all the repeated trials. Here, consider the assumption $\mathrm{A} 1$, then the state of the system is determined at all times even when the measurement does not perform on the system. Therefore, the values of joint probabilities of $Q_{1}, Q_{2}$ and $Q_{3}$ are determined a priori at initial time $t_{0}$. We denote it by the symbol $P_{i, j}\left(Q_{1}, Q_{2}, Q_{3}\right)$. Remark that the pairs of indexes $i, j$ encode the situation that only two observables $Q_{i}$ and $Q_{j}$ are measured. In other words, the joint probability depends on the situations which pairs of observables are measured. (We can consider pairs of indexes, instances of time, as parameters encoding three temporal contexts, $\mathcal{C}_{t_{1} t_{2}}, \mathcal{C}_{t_{1} t_{3}}, \mathcal{C}_{t_{2} t_{3}}$, cf. section 4) However if one considers (A2), then the joint probabilities do not depend on temporal context:3:

$$
P_{i, j}\left(Q_{1}, Q_{2}, Q_{3}\right)=P\left(Q_{1}, Q_{2}, Q_{3}\right) \quad \forall i, j
$$

Then we have the following equalities:

$$
\begin{aligned}
& P\left(Q_{1}, Q_{2}\right)=\sum_{Q_{3}= \pm 1} P\left(Q_{1}, Q_{2}, Q_{3}\right) \\
& P\left(Q_{2}, Q_{3}\right)=\sum_{Q_{1}= \pm 1} P\left(Q_{1}, Q_{2}, Q_{3}\right) \\
& P\left(Q_{1}, Q_{3}\right)=\sum_{Q_{2}= \pm 1} P\left(Q_{1}, Q_{2}, Q_{3}\right)
\end{aligned}
$$

which are consequences of the additivity of classical (Kolmogorov) probability. Thus pairwise joint probability distributions are context independent (as a consequence of (A2)). We also have

$$
\begin{aligned}
& P\left(Q_{1}\right)=\sum_{Q_{2}= \pm 1} P\left(Q_{1}, Q_{2}\right)=\sum_{Q_{3}= \pm 1} P\left(Q_{1}, Q_{3}\right) \\
& P\left(Q_{2}\right)=\sum_{Q_{1}= \pm 1} P\left(Q_{1}, Q_{2}\right)=\sum_{Q_{3}= \pm 1} P\left(Q_{2}, Q_{3}\right) \\
& P\left(Q_{3}\right)=\sum_{Q_{1}= \pm 1} P\left(Q_{1}, Q_{3}\right)=\sum_{Q_{2}= \pm 1} P\left(Q_{2}, Q_{3}\right)
\end{aligned}
$$

Thus, for each observable, its probability distribution is also context independent (as a consequence of (A2)). Violation of these equalities is interpreted as exhibition of contextuality. In psychology and cognitive science the equalities (1)-(3) represent the special case of so-called marginal selectivity 37- 39. It is clear that if at least one of these equalities is violated then one cannot assume existence of context independent joint probability distribution.

\footnotetext{
${ }^{3}$ We remark that conditions (A1) and (A2) were formulated in the physical framework. Therefore any study about the LG-inequality performed at the mathematical level of rigorousness has to present some mathematical formalization of these conditions. In our study (A1) and (A2) imply that there exists the joint probability distribution which does not depend on experimental contexts. In particular, we identify (A2) with noncontextuality. We understand well that this is not the only possible probabilistic interpretation of (A1) and (A2).
} 
Under the assumption of existence of the joint (triple) probability distribution the correlation functions are written with the joint probabilities $P\left(Q_{i}, Q_{j}\right)$ as

$$
\begin{aligned}
C_{i j} & =P\left(Q_{i}=1, Q_{j}=1\right)+P\left(Q_{i}=-1, Q_{j}=-1\right) \\
& -P\left(Q_{i}=-1, Q_{j}=1\right)-P\left(Q_{i}=1, Q_{j}=-1\right) \\
& =2\left\{P\left(Q_{i}=1, Q_{j}=1\right)+P\left(Q_{i}=-1, Q_{j}=-1\right)\right\}-1 .
\end{aligned}
$$

We set $K=C_{12}+C_{23}-C_{13}$. It can be represented in the following form:

$$
K=1-4\left\{P\left(Q_{1}=1, Q_{2}=-1, Q_{3}=1\right)+P\left(Q_{1}=-1, Q_{2}=1, Q_{3}=-1\right)\right\}
$$

This representation implies the LG-inequality:

$$
K \leq 1
$$

As we know, e.g., 13, 17, for the quantum correlation functions $C_{i j}$ the above inequality can be violated (theoretically and experimentally)

\section{Contextual LG inequality}

Here, we express the LG's assumptions in terms of context-dependent probabilities 9]. We remark that in general context-dependent probabilities cannot be represented in common Kolmogorov probability space. Therefore one can consider such contextual probabilistic models as nonKomogorovian probabilistic models, see the appendix.

(A1) There exists a joint probability $P_{\mathcal{C}}\left(Q_{1}, Q_{2}, Q_{3}\right)$ under a certain conditon of experiments (context) $\mathcal{C}$. And the Kolmogorovness of $P_{\mathcal{C}}\left(Q_{1}, Q_{2}, Q_{3}\right)$ is ensured within the context $\mathcal{C}$ :

$$
\begin{aligned}
& P_{\mathcal{C}}\left(Q_{1}, Q_{2}\right)=\sum_{Q_{3}= \pm 1} P_{\mathcal{C}}\left(Q_{1}, Q_{2}, Q_{3}\right) \\
& P_{\mathcal{C}}\left(Q_{2}, Q_{3}\right)=\sum_{Q_{1}= \pm 1} P_{\mathcal{C}}\left(Q_{1}, Q_{2}, Q_{3}\right) \\
& P_{\mathcal{C}}\left(Q_{1}, Q_{3}\right)=\sum_{Q_{2}= \pm 1} P_{\mathcal{C}}\left(Q_{1}, Q_{2}, Q_{3}\right)
\end{aligned}
$$

and

$$
\begin{aligned}
& P_{\mathcal{C}}\left(Q_{1}\right)=\sum_{Q_{2}= \pm 1} P_{\mathcal{C}}\left(Q_{1}, Q_{2}\right)=\sum_{Q_{3}= \pm 1} P_{\mathcal{C}}\left(Q_{1}, Q_{3}\right)=\sum_{Q_{2}= \pm 1} \sum_{Q_{3}= \pm 1} P_{\mathcal{C}}\left(Q_{1}, Q_{2}, Q_{3}\right), \\
& P_{\mathcal{C}}\left(Q_{2}\right)=\sum_{Q_{1}= \pm 1} P_{\mathcal{C}}\left(Q_{1}, Q_{2}\right)=\sum_{Q_{3}= \pm 1} P_{\mathcal{C}}\left(Q_{2}, Q_{3}\right)=\sum_{Q_{1}= \pm 1} \sum_{Q_{3}= \pm 1} P_{\mathcal{C}}\left(Q_{1}, Q_{2}, Q_{3}\right), \\
& P_{\mathcal{C}}\left(Q_{3}\right)=\sum_{Q_{2}= \pm 1} P_{\mathcal{C}}\left(Q_{2}, Q_{3}\right)=\sum_{Q_{1}= \pm 1} P_{\mathcal{C}}\left(Q_{1}, Q_{3}\right)=\sum_{Q_{1}= \pm 1} \sum_{Q_{2}= \pm 1} P_{\mathcal{C}}\left(Q_{1}, Q_{2}, Q_{3}\right) .
\end{aligned}
$$


(A2) Consider three different contexts $\mathcal{C}_{A}, \mathcal{C}_{B}$ and $\mathcal{C}_{C}$, then there exists a context $\mathcal{C}$ unifying the above contexts $\mathcal{C}_{A}, \mathcal{C}_{B}$ and $\mathcal{C}_{C}$ such that

$$
\begin{aligned}
& P_{\mathcal{C}_{A}}\left(Q_{1}, Q_{2}\right)=\sum_{Q_{3}= \pm 1} P_{\mathcal{C}}\left(Q_{1}, Q_{2}, Q_{3}\right), \\
& P_{\mathcal{C}_{B}}\left(Q_{2}, Q_{3}\right)=\sum_{Q_{1}= \pm 1} P_{\mathcal{C}}\left(Q_{1}, Q_{2}, Q_{3}\right), \\
& P_{\mathcal{C}_{C}}\left(Q_{1}, Q_{3}\right)=\sum_{Q_{2}= \pm 1} P_{\mathcal{C}}\left(Q_{1}, Q_{2}, Q_{3}\right) .
\end{aligned}
$$

From these assumptions, one can obtain the inequality (4) for $K$ given by

$K=1-4\left(P_{\mathcal{C}}\left(Q_{1}=1, Q_{2}=-1, Q_{3}=1\right)+P_{\mathcal{C}}\left(Q_{1}=-1, Q_{2}=1, Q_{3}=-1\right)\right)$.

\section{Violation of inequality in optical illu- sions}

The Schröder's stair is an ambiguous figure which induces optical illusion 16 . 42, see the Fig. 1. Our brain can switch between the two alternative interpretations of this figure:

(i) The surface of ' $\mathrm{L}$ ' is front, and the surface of ' $\mathrm{R}$ ' is back.

(ii) The surface of ' $R$ ' is front, and the surface of ' $L$ ' is back.

This switch-like process of human perception is called depth inversion, and many experimental proofs on this phenomenon have been reported. However, the details of its mechanism is not completely figured out even in recent studies.

It is well-known fact that the depth inversion depends on various contexts of figure; e.g. relative size of the surface ' $L$ ' for ' $R$ ', color or shadow in figure, angle to the horizon, etc. 42. Therefore we must define the contextual dependent probability that a person answers either (i) or (ii) in the experiment.

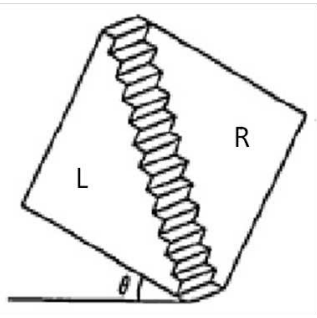

Figure 1: Schröder's stair leaning at angle $\theta$ 
Now we explain the method of our experiment, and we show its results. We show the subjects the picture of Schröder's stair which is leaning at a certain angle $\theta$ (see Fig. 1). We prepare the 11 pictures which are leaning at different angles: $\theta=0,10,20,30,40,45,50,60,70,80,90$. A subject must answer either (i) "L is front" or (ii) "R is front" for every picture. We arrange the computer experiment to change the pictures and to record their answers.

Before the experiment, we divided the subjects into three groups: (A) 55 persons, (B) 48 persons, (C) 48 persons 4 For the first group (A), the order of showing is randomly selected for each person. To assume statistically uniform randomness of this selection, we use the computerimplemented function (e.g. java.rand). For the second group (B), the angle $\theta$ is increased from a small value: $0,10, \cdots, 90$. Inversely, for the third group (C), the angle $\theta$ is decreased from a large value: $90,80, \cdots, 0$.

Thus we have the three kinds of experimental data: (A) the angle of Schröder stair changes randomly, (B) from 0 to 90 , (C) from 90 to 0. These contexs of experiments are denoted by $\mathcal{C}_{A}, \mathcal{C}_{B}$ and $\mathcal{C}_{C}$. Let $X_{\theta}$ be a random variable which takes \pm 1 . The event that a subject says "left side is front" corresponds to the result $X_{\theta}=+1$. Then, from the repeated trials for each experimental context, we have the experimentally obtained values of joint probabilities:

$P_{\mathcal{C}_{A}}\left(X_{0}, X_{10}, \cdots, X_{90}\right), \quad P_{\mathcal{C}_{B}}\left(X_{0}, X_{10}, \cdots, X_{90}\right), \quad P_{\mathcal{C}_{C}}\left(X_{0}, X_{10}, \cdots, X_{90}\right)$.

The correlation functions is given by

$$
\begin{aligned}
& C_{12}=2\left\{P_{\mathcal{X}}\left(X_{\theta_{1}}=1, X_{\theta_{2}}=1\right)+P_{\mathcal{X}}\left(X_{\theta_{1}}=-1, X_{\theta_{2}}=-1\right)\right\}-1, \\
& C_{23}=2\left\{P_{\mathcal{Y}}\left(X_{\theta_{2}}=1, X_{\theta_{3}}=1\right)+P_{\mathcal{Y}}\left(X_{\theta_{2}}=-1, X_{\theta_{3}}=-1\right)\right\}-1, \\
& C_{13}=2\left\{P_{\mathcal{Z}}\left(X_{\theta_{1}}=1, X_{\theta_{3}}=1\right)+P_{\mathcal{Z}}\left(X_{\theta_{1}}=-1, X_{\theta_{3}}=-1\right)\right\}-1 .
\end{aligned}
$$

Here, the triple $(\mathcal{X}, \mathcal{Y}, \mathcal{Z})$ is given by a combination of the contexts $\mathcal{C}_{A}, \mathcal{C}_{B}$ and $\mathcal{C}_{C}$. We show the values of $C_{12}, C_{23}$ and $C_{13}$ in the Table XX

\begin{tabular}{cccccccc} 
& $C_{12}$ & $C_{23}$ & $C_{13}$ & & $C_{12}$ & $C_{23}$ & $C_{13}$ \\
$\mathcal{C}_{A}$ & 1.000 & 0.964 & 0.964 & $\mathcal{C}_{A}$ & 0.091 & 0.091 & 0.127 \\
$\mathcal{C}_{B}$ & 0.917 & 0.833 & 0.750 & $\mathcal{C}_{B}$ & 0.375 & 0.625 & 0.083 \\
$\mathcal{C}_{C}$ & 0.917 & 1.000 & 1.000 & $\mathcal{C}_{C}$ & 0.625 & 0.375 & 0.167 \\
(Left) & $\left(\theta_{1}, \theta_{2}, \theta_{3}\right)=(0,10,20)$ & (Right) & \multicolumn{2}{c}{$\left(\theta_{1}, \theta_{2}, \theta_{3}\right)=(40,45,50)$}
\end{tabular}

We estimate the LHS of the inequality:

$$
K\left(\theta_{1}, \theta_{2}, \theta_{3}\right)=C_{12}+C_{23}-C_{13} .
$$

\footnotetext{
${ }^{4}$ We randomly selected the 151 participants from the students of Tokyo University of Science. And randomly divided this sample into three aforementioned groups. We showed a suite of 11 pictures to each subject with a laptop computer. Usually the distance between eyes and screen is $20 \mathrm{~cm} 30 \mathrm{~cm}$. He or she typed a key corresponding to answer $L$ or $R$. We did not limited time to answer, so that the subject had enough time to make his or her decision. We implemented java application in order to show the pictures and to record their answers. The order of showing in random case can be selected by software (not manually). All the records are saved as the output CSV file.
} 
Table YY shows that the value of $K$ with respect to the $\left(\theta_{1}, \theta_{2}, \theta_{3}\right)=$ $(0,10,20)$ and $(40,45,50)$. The value of $K$ exceeding one is seen in several cases.

$\begin{array}{cccccccccc}\mathcal{X}, \mathcal{Y}, \mathcal{Z} & \mathcal{C}_{A}, \mathcal{C}_{A} & \mathcal{C}_{A}, \mathcal{C}_{B} & \mathcal{C}_{A}, \mathcal{C}_{C} & \mathcal{C}_{B}, \mathcal{C}_{A} & \mathcal{C}_{B}, \mathcal{C}_{B} & \mathcal{C}_{B}, \mathcal{C}_{C} & \mathcal{C}_{C}, \mathcal{C}_{A} & \mathcal{C}_{C}, \mathcal{C}_{B} & \mathcal{C}_{C}, \mathcal{C}_{C} \\ \mathcal{C}_{A} & 1.000 & 1.214 & 1.047 & 0.870 & 1.083 & 0.917 & 1.036 & 1.250 & 1.083 \\ \mathcal{C}_{B} & 0.917 & 1.130 & 0.964 & 0.786 & 1.000 & 0.833 & 0.953 & 1.167 & 1.000 \\ \mathcal{C}_{C} & 0.917 & 1.130 & 0.964 & 0.786 & 1.000 & 0.833 & 0.953 & 1.167 & 1.000\end{array}$

Table 1: the triple of angles $(0,10,20)$. The values of $K$ for various combinations of contexts. For the contexts $\left(\mathcal{C}_{A}, \mathcal{C}_{C}, \mathcal{C}_{B}\right), K$ approaches its maximal value.

$\begin{array}{cccccccccc}\mathcal{X}, \mathcal{Y}, \mathcal{Z} & \mathcal{C}_{A}, \mathcal{C}_{A} & \mathcal{C}_{A}, \mathcal{C}_{B} & \mathcal{C}_{A}, \mathcal{C}_{C} & \mathcal{C}_{B}, \mathcal{C}_{A} & \mathcal{C}_{B}, \mathcal{C}_{B} & \mathcal{C}_{B}, \mathcal{C}_{C} & \mathcal{C}_{C}, \mathcal{C}_{A} & \mathcal{C}_{C}, \mathcal{C}_{B} & \mathcal{C}_{C}, \mathcal{C}_{C} \\ \mathcal{C}_{A} & 0.055 & 0.099 & 0.015 & 0.589 & 0.633 & 0.549 & 0.339 & 0.383 & 0.299 \\ \mathcal{C}_{B} & 0.339 & 0.383 & 0.299 & 0.873 & 0.917 & 0.833 & 0.623 & 0.667 & 0.583 \\ \mathcal{C}_{C} & 0.589 & 0.633 & 0.549 & 1.123 & 1.167 & 1.083 & 0.873 & 0.917 & 0.833\end{array}$

Table 2: the triple of angles $(40,45,50)$. The values of $K$ for various combinations of contexts. For the contexts $\left(\mathcal{C}_{C}, \mathcal{C}_{B}, \mathcal{C}_{B}\right), K$ approaches its maximal value.

\section{$6 \quad$ Statistical analysis}

We start from the random variable:

$$
K=Q_{1} Q_{2}+Q_{2} Q_{3}-Q_{1} Q_{3}
$$

Here, $K$ takes $-4,-2,0$ or +3 since $Q_{i}$ takes +1 or -1 . The probability distribution of $K$ is not known, but it has mean value $\mu$ and variance $\sigma^{2}$ and their statistical estimates can be found. To find the confidence interval in such a situation, we apply the simplest method of nonparametric statistics, namely, the method based on the Chebyshev inequality. (However, from the very beginning we remark that this method gives us only rough estimate for the confidence interval.) This method was recently used 43. for analysis of statistical data from the Vienna-test for the Bell-type inequality, the Eberhard inequality, which finally closed the fair sampling loophole. In this test, because of the presence of slight drift depending on experimental setting, one cannot assume Gaussianity of data and it seems that usage of the Chebyshev inequality is the simplest way to resolve this problem.

We can apply the Chebyshev inequality to sample mean of $K$

$$
P(|m-\mu|>c) \leq \frac{\sigma^{2}}{n c^{2}}
$$

with positive constant $c$. Here, $m$ is a sample mean of independent random variables $K_{1}, \ldots, K_{n}$ :

$$
m=\frac{K_{1}+K_{2}+\ldots+K_{n}}{n}
$$


Although we do not know the value of $\sigma^{2}$, we can estimate $\sigma^{2}$ with unbiased sample variance. Then $\mu$ is estimated by $m$ with confidence interval $[m-c, m+c]$.

We take the $80 \%$ confidence level. In the case that the order of the contexts is $(\mathcal{X}, \mathcal{Y}, \mathcal{Z})=\left(\mathcal{C}_{A}, \mathcal{C}_{C}, \mathcal{C}_{B}\right)$, and the angle $\theta=0,10,20$ (This case is maximum value of $K$ ), we estimate the value of $K$ as follows.

$$
K=1.250 \pm 0.213
$$

Statistical analysis shows that the violation of the LG-inequality is statistically significant. However, it is clear that one has to perform better experiments to get a higher level of violation. The main problem of the present experiment is that the sample is not large enough. It seems that this will be the problem of all Bell-type tests in cognitive science. All such inequalities are based on correlations, so samples of students have to be sufficiently large: to calculate correlations (this needs human recourses)and get statistically significant violation (additional human recourse) of the corresponding inequality for such correlations.

\section{Concluding remarks}

A violation of the contextual LG inequality by statistical data collected for observations of the Schröder stair rotated for different angles supports the contextual cognition paradigm presented in the series of works [], 19][24], 14. Our experimental statistical data is fundamentally contextual 5 The brain does not have a priori prepared "answers" to the question about the $\mathrm{R} / \mathrm{L}$ structure of the Schröder stair for the fixed angle $\theta$. Answers are generated depending on the mental context. Thus mental realism is a kind of contextual realism, cf. [30]. There are practically no (at least not so many), so to say, "absolute mental quantities", "answers" to the same question vary essentially depending on context. This conclusion is not surprising in the framework of cognitive science and psychology, where various framing effects are well known. Thus the main contribution of this paper is the demonstration of applicability of a statistical test of

\footnotetext{
${ }^{5}$ In fact, it violates even condition of marginal selectivity. We interpret violation of marginal selectivity as one of signs of contextuality. Of course, it is important to approach violation of the LG-inequality in combination with marginal selectivity. And this is a delicate issue. Up to authors' knowledge, even in physics the experimental situation is not completely clear. Practically all publications on violation of the Bell-type inequalities represent only the values of correlations (or even only the values of their linear combinations) and it is impossible to check condition of marginal selectivity. Only two authors, A. Aspect (in his PhD-thesis 45]) and G. Weihs (open source, which was later removed), presented count-data for which it is possible to check marginal selectivity. And (surprisingly) this condition is violated 46. Thus the question whether in physics combination of violation of the Bell-type inequalities and marginal selectivity was approached is open (mainly because experimental groups do not want to share count-data). We can speculate that in physics marginal selectivity would be never approached, at least in combination with violation of the Bell-type inequalities. Marginal distributions would always depend on experimental settings. However, the degree of such dependence can be considered as "sufficiently small comparing with the degree of violation of the corresponding inequality." Of course, the latter statement has to be presented in terms of statistical analysis.
} 
contextuality borrowed from quantum physics. We also can consider this study as a step towards creation of unified mathematical picture of the world: physical and mental phenomena can be described by the same equations, cf. [6].

Such studies on usage of the standard quantum mechanical tests in other domains of science also contribute to foundations of quantum physics, since they can be used as at least indirect arguments supporting some interpretations of outputs of these tests for physical systems. In our case, the viewpoint that contextuality and not nonlocality is the basic source of nonclassical probabilistic behavior of cognitive systems, see also 44, may be used to support local contextual models of quantum physical phenomena.

\section{Appendix: Kolmogorov probability model and Bell type inequalities}

\subsection{Kolmogorov axiomatics, probability space}

We start this section with rather long introduction to the classical measuretheoretic model of probability theory 18 . Our aim is to show that the problem of construction of common probability measure was one of the basic problems even in classical probability theory, namely, invention of stochastic processes was based on such a special construction (Kolmogorov's theorem [18). Positive solution of this problem in the case of stochastic processes played an important role in the formation of the present ideology of classical probability: observables have to be represented by random variables on common probability space. However, in the case of quantum observables this is not true.

Classical probability theory is based on the model of A. N. Kolmogorov [18. Its basic notion is probability space, a triple $\mathcal{P}=(\Omega, \mathcal{F}, P)$, where $\Omega$ is a set, $P$ is a probability measure, and $\mathcal{F}$ is a collection of subsets of $\Omega$ on which probability is defined 6 In the Kolmogorovean model [18] an observable, say $a$, is represented by a random variabl $7:$ a map $a: \Omega \rightarrow \mathbf{R}$ such that, for each interval, its pre-image, $\{\omega \in \Omega: a(\omega) \in[\alpha, \beta)\}$, belongs to $\mathcal{F}$. Its the probability distribution is defined as $p_{a}(A)=P(\omega: a(\omega) \in$ $A)$.

A system of observables is represented by a vector of random variables $a=\left(a_{1}, \ldots, a_{n}\right)$. Its probability distribution is defined as

$$
p_{a}\left(A_{1} \times \cdots \times A_{n}\right)=P\left(\omega: a_{1}(\omega) \in A_{1}, \cdots, a_{n}(\omega) \in A_{n}\right) .
$$

\footnotetext{
${ }^{6}$ This is a $\sigma$-algebra (" $\sigma$-field"): a system of sets which is closed with respect to countable unions, intersections, and the operation of complement.

${ }^{7}$ We remark that the Kolmogorovean model is not simply a mathematical theory. In the same way as the Euclidean model provided mathematical formalization of geometry of physical space, the Kolmogorovean model provided mathematical formalization of the theory of measurements of random variables. Euclid formalized such heuristic notions as point, straight line, plane, angle...; Kolmogorov formalized such notions as event, probability, random observable (=random variable). In section 8.3 we shall come back to comparison of the roles played by the Euclidean model of geometry of space and the Kolmogorovean model of random measurements in physics.
} 
The notion of a random vector is generalized to the notion of a stochastic process. Suppose that the set of indexes is infinite; for example, $a_{t}, t \in[0,+\infty)$. Suppose that, for each finite set $\left(t_{1} \ldots t_{k}\right)$, the vector $\left(a_{t_{1}} \ldots a_{t_{k}}\right)$ can be observed and its probability distribution $p_{t_{1} \ldots t_{k}}$ is given. By selecting $\Omega_{t_{1} \ldots t_{k}}=\mathbf{R}^{k}, P_{t_{1} \ldots t_{k}}=p_{t_{1} \ldots t_{k}}$, and $\mathcal{F}$ as the Borel $\sigma$-algebra, we obtain the probability space $\mathcal{P}_{t_{1} \ldots t_{k}}$ describing measurements at points $t_{1} \ldots t_{k}$. At the beginning of 20th century the main mathematical question of probability theory was whether it is possible to find a single probability space $\mathcal{P}=(\Omega, \mathcal{F}, P)$ such that all $a_{t}$ be represented as random variables on this space and all probability distributions $p_{t_{1} \ldots t_{k}}$ are induced by the same $P$ :

$$
p_{t_{1} \ldots t_{k}}\left(A_{1} \times \cdots \times A_{k}\right)=P\left(\omega \in \Omega: a_{t_{1}}(\omega) \in A_{1}, a_{t_{n}}(\omega) \in A_{n}\right) .
$$

Kolmogorov found natural conditions for the system of measures $p_{t_{1} \ldots t_{k}}$ which guarantee existence of such a probability space, see 18, And during the next 80 years analysis of properties of (finite and infinite) families of random variables defined on one fixed probability space was the main activity in probability theory.

\subsection{Kolmogorovean formalization of Bell's argu- ment}

Although J. Bell did not formulate his argument in the terms of a probability space, the problem of local realism for quantum observables was formalized in complete accordance with classical probability theory. In the Bell framework $\Omega$ is selected as the set of hidden variables $\Lambda$; local realism is equivalent to mathematical presentation of observables by random variables $\lambda \rightarrow a_{\alpha}(\lambda), b_{\beta}(\lambda)$. Here $a_{\alpha}, \alpha=\alpha_{1}, \alpha_{2}$, are observables depending on the parameter $\alpha$, experimental settings, at "Alice's lab" and $b_{\beta}, \beta=\beta_{1}, \beta_{2}$, are observables depending on the parameter $\beta$, experimental settings, at "Bob's lab". In the framework of the Kolmogorov probability model the Bell inequality is a theorem. A. Fine rigorously proved [4] that the Bell inequality is satisfied iff the common probability space for random variables representing quantum observables does exist.

In the LG inequality (which a special form of contextual Bell inequalities) there is a single observable $a_{t}$ depending on the time parameter. Kolmogorovness means that all these observables can be represented by random variables, $\lambda \rightarrow a_{t}(\lambda)$, on common Kolmogorov probability space.

\subsection{Models: (non-)Euclidean geometry and (non-)Kolmogorovean probability}

Although the main stream in classical probability was, so to say, the "common probability space stream", we can point to a few attempts to swim against this stream, e.g., [28, [9], 29], 30] 20], 15], 31]-39].

\footnotetext{
${ }^{8}$ We just remark that the $\Omega$ is selected as the set of all trajectories $t \rightarrow \omega(t)$. The random variable $a_{t}$ is defined as $a_{t}(\omega)=\omega(t)$. Construction of the probability measure $P$ serving for all finite random vectors is mathematically advanced and going back to construction of the Wiener measure on the space of continuous functions.
} 
Now we come back to comparison of mathematical formalizations of geometry of physical space and random observations. Since the work of Lobachevsky (and Gauss and Boyai), mathematicians understood that there exist different mathematical possibilities for representation of space geometry. We remark that already Lobachevsky and Gauss studied the problem of adequacy of the Euclidean model to physical reality. Lobachevsky proposed some astronomic tests, Euclidean contra Lobachevsky geometries; in Germany Gauss (who had some administrative obligations to measurements of land) performed measurement of angles of a huge triangle formed by three mountains. The latter test confirmed that at least locally we live in Euclidean space. Later Riemann formulated the general principles of geometry which played the fundamental role in mathematical representation of Einstein's general relativity. (Lobachesky geometry was used in special relativity).

We emphasize that mathematicians understood long before physicists (who were at that time completely busy with Newtonian physics based on the Euclidean geometry) that the Euclidean geometry is one of possible models of space. On the basis of such an experience collected in the mathematical community physicists were not astonished by the appearance of Minkowski space in special relativity and then (pseudo-)Riemann space in general relativity. Here the general approach of D. Hilbert on axiomatization of physics (also known as Hilbert's sixth problem) was respected.

Development of mathematical formalization of probability was very different. Mathematicians (with a few exceptions) did not question the Kolmogorov axiomatics. The first non-Kolmogorovean model was elaborated in physics as a part of new physical theory - quantum mechanics. And in classical probability community quantum probability is still not recognized as a probability theory, but as some exercises in noncommutative algebra. Therefore in probability it is more difficult than it was in the case of geometry: any mathematical model, including the Kolmogorovean model, has a restricted domain of application. Quantum phenomena simply showed that one special model of probability cannot be applied. From the viewpoint of Hilbert axiomatization of physics this is the end of the story, i.e., one need not search additional "explanations" of non-Kolmogorovness, one simply has to find a new appropriate mathematical model of probability and proceed with such a model. Thus from such a viewpoint the Bell argument is not about locality and realism, but about inadequacy of the Kolmogorov model 9 (Mathematical foundation of non-Kolmogorov probability theory was discussed in the books [9], 40, [8.)

Let us again make a comparison with geometry. Did Einstein try to "explain" appearance of (pseudo-)Riemannian geometries in general

\footnotetext{
${ }^{9}$ We point out that one of the problems slowing clarification of Bell's argument is that here typically probability (both classical and quantum) is not treated in the axiomatic framework. There is a prejudice that "probability is probability" and it can be understood heurictically without going to mathematical axiomatization. However, the positive experience of physical applications of the axiomatic mathematical models of geometry tells us that this is the most fruitful way even for probabilistic applications. (Nowadays in physics nobody would work in the framework of "heuristically understandable geometry".)
} 
relativity, instead of one special geometry (Euclidean)? Not at all, he simply identified (pseudo-)Riemannian geometry with physical space.

Thus experimental tests of Bell's inequality can be considered as tests of adequacy of the traditional Kolmogorov model to quantum physical phenomena (cf. with aforementioned Gauss test of the Euclidean model).

Typically adherents of the non-Kolmogorovness viewpoint on violation of the Bell inequality consider physics (in the spirit of the Hilbert program of axiomatization of physics) as a collection of mathematical models formalizing various natural phenomena. From the very beginning it is assumed that any such a model has a restricted domain of applications. A physical experiment of which the output cannot be described by a model under application is considered as a signal for creation of a new mathematical model, e.g., the Euclidean model could not be used for special relativity and new non-Euclidean models were explored (the Lobachevsky in special relativity and pseudo-Riemannian geometries in general relativity.)

\subsection{Reconstructing Kolmogorovness from contex- tuality}

The main message of this paper is that experimental statistical data collected in cognitive science and psychology are fundamentally contextual. In general for each experimental context data is described by its own Kolmogorov probability space 10

\section{Is it possible to unify these spaces in some way?}

One possibility is to use quantum probability and the formalism of complex Hilbert space. This is so to say nonclassical unification. Surprisingly it is even possible to unify these probability spaces classically, i.e., to embed them into single "big Kolmogorov space"! Such classical unification is based on taking into account randomness of realizations of contexts. In this "big probability space" the original probabilities appear as conditional probabilities with respect to various contexts.

The first version of such unification was presented in the paper 48 (see 49] for better structured presentation), where the probabilistic data collected in experiments to determine the EPR-Bohm correlations were

\footnotetext{
${ }^{10}$ We remark that this was the original viewpoint of Kolmogorov. In second section of his seminal monograph 18 in which he formulated the axiomatics of classical probability theory he emphasized the correspondence: experiment $\rightarrow$ probability space. Unfortunately, this message of the creator of probability theory was completely forgotten by his followers who completely ignored multi-space structure of experimental studies, see [53] for the detailed discussion. Of course, Kolmogorov by himself was excited by the possibility to unify probability spaces corresponding to measurements of a stochastic process for finite sequences of instances of time, see section 8.1 One cannot exclude that he was sure that such unification is possible for any kind of data. Although he had never pointed to such a possibility, some indirect signs supporting such a hypothesis about his views can be found; in particular, people from his close circle reacted very negatively to the attempt of Vorobj'ev [54] to proceed with multi-space approach in a series of applications - game theory, optimization theory. We remark that, in particular, Vorobj'ev 54] derived (all possible) Bell type inequalities (for any number of variables yielding any fixed number of values). He used these inequalities as tests of Kolmogorovness of probabilistic data.
} 
embedded into single Kolmogorov probability space. (This construction is evidently generalized to statistical data collected for any family of experimental contexts.) 11

The reader may ask: Why do you emphasize non-Kolmogorovness in such a situation?

Although "big Kolmogorov space" unifying data collected for different contexts exists, it cannot be used so successfully as quantum probability. The latter describes all possible experimental contexts homogeneously. In the classical approach based on reconstruction of Kolmogorovness from contextuality one has to take into account randomness of realizations of concrete contexts. Of course, one can construct huge Kolmogorov space unifying all possible contexts and all possible types of randomness for them. However, it would be practically impossible to work with such space.

Another problem of the Kolmogorovian unification of contextual experimental data is that by taking into account randomness of realizations of contexts (e.g., for the Bell-type experiments, how often each pair of orientations of polarization beam splitters is realized in the concrete experiment) we lost the internal description of data: experimenter's "free will" (to use this or that experimental context for the next trial) also has to be taken into account. This is a complex interpretation problem related to construction of "big Kolmogorov spaces", see 49] for discussion. Quantum mechanics provides description of experimental probabilities without taking into account randomness of realizations of experimental contexts; in this sense the quantum description can be treated as a kind of intrinsic description. And data can be "intrinsically" Kolmogorovian or nonKolmogorovian (although, as we emphasized in this section, it is always possible to make these data Kolmogorovian "externally").

Acknowledgment: We would like to thank H. Atmanspacher, I. Basieva, N. Watanabe, and I. Yamato for discussions. This work was partially supported by MPNS COST Action MP1006 (Fundamental Problems in Quantum Physics) and visiting fellowships (A. Khrennikov) to Center of Quantum BioInformatics, Tokyo University of Science and Institute for Quantum Optics and Quantum Information, Austrian Academy of Sciences.

\section{References}

[1] E. Conte, O. Todarello, A. Federici, F. Vitiello, M. Lopane, A. Khrennikov, A preliminary evidence of quantum-like behaviour in measurements of mental states. Proc. Int. Conf. Quantum Theory: Reconsideration of Foundations. Ser. Math. Modelling in Phys., Engin., and Cogn. Sc., 10, 679-702, Växjö Univ. Press, 2004.

[2] E. Conte, O. Todarello, A. Federici, F. Vitiello, M. Lopane, A. Khrennikov and J. P. Zbilut, Some remarks on an experiment suggesting quantum-like behavior of cognitive entities and formulation

\footnotetext{
${ }^{11}$ See also recent studies $[50-[52]$.
} 
of an abstract quantum mechanical formalism to describe cognitive entity and its dynamics. Chaos, Solitons and Fractals, 31 (5), 10761088 (2007).

[3] Conte, E., Khrennikov, A., Todarello, O., Federici, A., Mendolicchio, L, Zbilut, J. P.: Mental state follow quantum mechanics during persception and cognition of ambiguous figures. Open Systems and Information Dynamics, 16, 1-17 (2009).

[4] E. Conte, A. Khrennikov, O. Todarello, et al., A preliminary experimental verification on the possibility of Bell inequality violation in mental states. Neuroquantology, 6, N 3, 214-221 (2008).

[5] J. Bell, Speakable and Unspeakable in Quantum Mechanics. Cambridge Univ. Press, Cambridge (1987).

[6] A. Khrennikov, Ubiquitous quantum structure: from psychology to finances, Springer, Berlin-Heidelberg-New York, 2010.

[7] J. R. Busemeyer and P. D. Bruza, Quantum models of cognition and decision. Cambridge Press, (2012).

[8] Asano, M., Khrennikov, A., Ohya, M., Tanaka, Y., Yamato, I. (2014) Quantum Adaptivity in Biology: from Genetics to Cognition. Springer, Berlin-Heidelberg, to be published.

[9] A. Khrennikov, Contextual approach to quantum formalism, Springer, Berlin-Heidelberg-New York, 2009.

[10] H. Atmanspacher, Th. Filk, and H. Römer, (2004). Quantum Zeno features of bistable perception. Biological Cybernetics, 90, 33-40.

[11] H. Atmanspacher and Th. Filk, Temporal nonlocality in bistable perception In: Quantum Theory: Reconsiderations of Foundations - 6, A. Khrennikov, H. Atmanspacher, A. Migdall and S. Polyakov (eds.), Special Section: Quantum-like decision making: from biology to behavioral economics, AIP Conf. Proc. 1508, (2012), pp. 79-88.

[12] Atmanspacher, H., Filk, T. and Römer, H. Weak quantum theory: Formal framework and selected applications. In: Adenier, G., Khrennikov, A., and Nieuwenhuizen, T.M. (eds.), Quantum Theory: Reconsideration of Foundations 3. American Institute of Physics, New York, (2006) pp. 34-46.

[13] A. Leggett and A. Garg, PRL 54, 857-860 (1985).

[14] M. Asano, T. Hashimoto, A. Khrennikov, M. Ohya, Y. Tanaka, Adaptive dynamics and optical illusion on Schröder's stair. In: Quantum Interactions 13, University of Leicester Press, Leicester 2013.

[15] E. N. Dzhafarov and J. V. Kujala, Quantum entanglement and the issue of selective influences in psychology: An overview. Lecture Notes in Computer Science 7620, 184-195 (2012).

[16] H.Schröder, "Uber eine optische Inversion bei Betrachtung verkehrter, durch optische Vorrichtung entworfener physischer Bilder", Annalen der Physik, 181(10). 298-311 (1858) 
[17] M. E. Goggin, M. P. Almeida, M. Barbieri, B. P. Lanyon, J. L. O'Briend, A. G. White, and G. J. Pryde, Violation of the LeggettGarg inequality with weak measurements of photons. Proc. Nat. Acad. Sc. 108, N 4, 12561261 (2010).

[18] A. N. Kolmogoroff, Grundbegriffe der Wahrscheinlichkeitsrechnung. Springer Verlag, Berlin (1933); English translation: A. N. Kolmogorov, Foundations of Theory of Probability. Chelsea Publishing Company, New York (1956).

[19] M. Asano, I. Basieva, A. Khrennikov, M. Ohya and I. Yamato, NonKolmogorovian approach to the context-dependent systems breaking the classical probability law. Found. Phys. 43, 895-911 (2013).

[20] Khrennikov, A. (2010). Classical and quantum probability for biologists-introduction. QP - PQ: Quantum Probability and White Noise Analysis. 26. 179-192.

[21] Asano, M., Ohya, M., Khrennikov, A. (2011). Quantum-Like Model for Decision Making Process in Two Players Game : A NonKolmogorovian Model. Foundations of physics. 41. 538-548.

[22] Asano, M., Masanori, O., Tanaka, Y., Basieva, I., Khrennikov, A. (2011). Quantum-like model of brain's functioning: Decision making from decoherence. Journal of Theoretical Biology. 281. 56-64.

[23] Asano, M., Basieva, I., Khrennikov, A., Ohya, M., Tanaka, Y. (2012). Quantum-like dynamics of decision-making. Physica A : Statistical Mechanics and its Applications. 391. 2083-2099.

[24] Asano, M., Basieva, I., Khrennikov, A., Ohya, M., Tanaka, Y. (2012). Quantum-like Dynamics of Decision-making in Prisoner's Dilemma Game. AIP Conference Proceedings. 1424. 453-457.

[25] Asano, M., Basieva, I., Khrennikov, A., Ohya, M., Tanaka, Y., et al. (2012). Quantum-like model of diauxie in Escherichia coli : operational description of precultivation effect. J. Theor. Biol. 314. 130-137.

[26] Asano, M., Basieva, I., Khrennikov, A., Ohya, M., Tanaka, Y., Yamato, I. (2012). Quantum-like model of glucose effect on Escherichia coli growth. AIP Conference Proceedings. 1424. 507-512.

[27] H. Bartosik, J. Klepp, C. Schmitzer, S. Sponar, A. Cabello, H. Rauch and Y. Hasegawa, Experimental test of quantum contextuality in neutron interferometry. Phys. Rev. Lett. 103, 040403 (2009).

[28] Khrennikov, A. (2008). Bell-Boole inequality: Nonlocality or probabilistic incompatibility of random variables?. Entropy. 10. 19-32.

[29] A. Khrennikov, Violation of Bell's Inequality and Postulate on Simultaneous Measurement of Compatible Observables. J. Computational and Theoretical Nanoscience 8, N 6, 1006-1010 (2011).

[30] A. Khrennikov Bell argument: Locality or Realism? Time to make the choice. AIP Conference Proceedings. 1424. 160-175 (2012); arXiv:1108.0001 [quant-ph]

[31] Accardi, L.: Topics in quantum probability. Phys. Rep. 71, 169192 (1981). 
[32] M. Kupczynski, Phys. Lett. A 121, 205 (1987).

[33] K. Hess and W. Philipp, Critique of Proofs, with and without Inequalities. In: Foundations of Probability and Physics-3, AIP Conf. Proc., vol. 750, ed. A. Khrennikov, Melville, AIP, 2005, pp. 516523.

[34] Th. M. Nieuwenhuizen, Where Bell went wrong? AIP Conf. Proc. 1101: Foundations of Probability and Physics - 5; Luigi Accardi, Guillaume Adenier, Christopher A. Fuchs, Gregg Jaeger, A. Khrennikov, J.-A. Larsson, and S. Stenholm, eds, (Am. Inst. Phys., Melville, NY, 2009), pp 127-133.

[35] T. M. Nieuwenhuizen Is the Contextuality Loophole Fatal for the Derivation of Bell Inequalities? Found. Phys. 41, 580-591 (2011)

[36] K. Michielsen, F. Jin, M. Delina and H. De Raedt, Event-by-event simulation of nonclassical effects in two-photon interference experiments In: FQMT'11: Frontiers of Quantum and Mesoscopic Thermodynamics (Prague, Czech Republic, 25-30 July 2011); T. M. Nieuwenhuizen, P. D, Keefe and V. Spicka (eds.) Physica Scripta, T151, Article Number: 014005 (2012).

[37] Dzhafarov, E.N. (2003). Selective influence through conditional independence. Psychometrika, 68, 7-26.

[38] Dzhafarov, E.N., and Kujala, J.V. (2014). On selective influences, marginal selectivity, and Bell/CHSH inequalities. Topics in Cognitive Science 6, 121128.

[39] Dzhafarov, E.N., and Kujala, J.V. Probability, random variables, and selectivity. arXiv:1312.2239

[40] Ohya. M., Volovich I. V.: Mathematical Foundations of Quantum Information and Computation and Its Applications to Nano- and Bio-systems, Springer, Berlin-New York (2011).

[41] Bohr, N.: The philosophical writings of Niels Bohr, 3 vols. Woodbridge, Conn., Ox Bow Press (1987)

[42] M. Ohya, "Optical illusions in view of phase transition ", SuriKagaku (Japanese). 301. 58-61 (1988)

[43] A. Khrennikov, S. Ramelow, R. Ursin, B. Wittmann, J. Kofler, I. Basieva, On the equivalence of Clauser-Horne and Eberhard inequality based tests. This special issue of Physica Scripta.

[44] J. Acacio de Barros, Joint probabilities and quantum cognition, In: Quantum Theory: Reconsiderations of Foundations - 6, A. Khrennikov, H. Atmanspacher, A. Migdall and S. Polyakov (eds.), Special Section: Quantum-like decision making: from biology to behavioral economics, AIP Conf. Proc. 1508, (2012), pp. 98-104.

[45] A. Aspect, Three experimental tests of Bell inequalities by the measurement of polarization correlations between photons. Orsay (1983).

[46] Adenier, G. and Khrennikov, A. Yu.: Is the fair sampling assumption supported by EPR experiments? Phys. B: Atomic, Molecular and Optical Physics 40 (1), 131-141 (2007) 
[47] A. Fine, Found. Phys. 19, N 5, 453-462 (1989).

[48] D. Avis, P. Fischer, A. Hilbert, A. Khrennikov, "Single, Complete, Probability Spaces Consistent With EPR-Bohm-Bell Experimental Data", in: Foundations of Probability and Physics-5, AIP Conference Proceedings, Volume 750, ed. Andrei Khrennikov,Melville,New York,AIP,2009, pp. 294-301.

[49] A. Khrennikov, Classical probability model for Bell inequality. Proc. EmQM13: Emergent Quantum Mechanics 2013, 36 October 2013, Vienna, Austria. J. Physics: Ser. Conf. Proc., 504, art. N 012019 (2014).

[50] Dzhafarov, E.N., and Kujala, J.V. (2014). Embedding quantum into classical: contextualization vs conditionalization. PLoS ONE 9(3): e92818. doi:10.1371/journal.pone.0092818.

[51] Dzhafarov, E.N., and Kujala, J.V. (2014). No-Forcing and NoMatching theorems for classical probability applied to quantum mechanics. Foundations of Physics 44, 248-265.

[52] Dzhafarov, E.N., and Kujala, J.V. (in press). A qualified Kolmogorovian account of probabilistic contextuality. Lecture Notes in Computer Science (to be published).

[53] Khrennikov, Interpretations of Probability. De Gruyter, Berlin, 2009, second edition (completed).

[54] Vorob'ev, N. N., Consistent families of measures and their extensions. Theory of Probability and its Applications 7, 147-162 (1962) 